\title{
Infinitesimal Deformations and Brauer Group of Some Generalized Calabi-Eckmann Manifolds
}

\author{
Indranil BISWAS, Mahan MJ* and Ajay Singh THAKUR \\ Tata Institute of Fundamental Research and RKM Vivekananda University* \\ (Communicated by N. Suwa)
}

\begin{abstract}
Let $X$ be a compact connected Riemann surface. Let $\xi_{1}: E_{1} \longrightarrow X$ and $\xi_{2}: E_{2} \longrightarrow X$ be holomorphic vector bundles of rank at least two. Given these together with a $\lambda \in \mathbf{C}$ with positive imaginary part, we construct a holomorphic fiber bundle $S_{\lambda}^{\xi_{1}, \xi_{2}}$ over $X$ whose fibers are the Calabi-Eckmann manifolds. We compute the Picard group of the total space of $S_{\lambda}^{\xi_{1}, \xi_{2}}$. We also compute the infinitesimal deformations of the total space of $S_{\lambda}^{\xi_{1}, \xi_{2}}$. The cohomological Brauer group of $S_{\lambda}^{\xi_{1}, \xi_{2}}$ is shown to be zero. In particular, the Brauer group of $S_{\lambda}^{\xi_{1}, \xi_{2}}$ vanishes.
\end{abstract}

\section{Introduction}

Let $X$ be compact connected Riemann surface. Let $\xi_{1}: E_{1} \longrightarrow X$ and $\xi_{2}: E_{2} \longrightarrow X$ be holomorphic vector bundles of rank $m$ and $n$ respectively, with $m, n \geq 2$. Let $E_{1}^{0}$ (respectively, $E_{2}^{0}$ ) be the complement of the image of the zero section in $E_{1}$ (respectively, $E_{2}$ ). Fix a complex number $\lambda$ with positive imaginary part.

The group $\mathbf{C}$ acts on the fiber product $E_{1}^{0} \times{ }_{X} E_{2}^{0}$ as follows:

$$
t \cdot(z, w)=(\exp (t) \cdot z, \exp (t(\lambda-1) / \lambda) \cdot w), \quad t \in \mathbf{C},(z, w) \in E_{1}^{0} \times_{X} E_{2}^{0} .
$$

The quotient for this action is a compact complex manifold; we denote this complex manifold by $S_{\lambda}^{\xi_{1}, \xi_{2}}$. Each fiber of the natural projection $p: S_{\lambda}^{\xi_{1}, \xi_{2}} \longrightarrow X$ is a Calabi-Eckmann manifold.

Define the elliptic curve $T:=\mathbf{C} /(\mathbf{Z} \oplus \lambda \cdot \mathbf{Z})$.

We prove the following (see Theorem 3.6 and Corollary 5.2):

THEOREM 1.1. The Picard group of $S_{\lambda}^{\xi_{1}, \xi_{2}}$ fits in a short exact sequence

$$
0 \longrightarrow \operatorname{Pic}(X) \longrightarrow \operatorname{Pic}\left(S_{\lambda}^{\xi_{1}, \xi_{2}}\right) \longrightarrow H^{1}\left(T, \mathcal{O}_{T}\right) \longrightarrow 0
$$

Received November 27, 2012; revised May 10, 2013

2000 Mathematics Subject Classification: 14F22, 32Q55, 32G05

Key words and phrases: Calabi-Eckmann manifold, infinitesimal deformation, Brauer group, Borel spectral sequence 
The injective homomorphism $\operatorname{Pic}(X) \longrightarrow \operatorname{Pic}\left(S_{\lambda}^{\xi_{1}, \xi_{2}}\right)$ sends any holomorphic line bundle $L$ to its pullback $p^{*} L$.

THEOREM 1.2. The cohomological Brauer group $\operatorname{Br}^{\prime}\left(S_{\lambda}^{\xi_{1}, \xi_{2}}\right)$ vanishes. In particular, the Brauer group $\operatorname{Br}\left(S_{\lambda}^{\xi_{1}, \xi_{2}}\right)$ vanishes.

Assume that all endomorphisms of the holomorphic vector bundles $E_{1}$ and $E_{2}$ are scalar multiplications. Also, assume that the genus of $X$ is at least two. We prove the following (see Corollary 4.4):

THEOREM 1.3. The dimension of the space of all infinitesimal deformations of the complex manifold $S_{\lambda}^{\xi_{1}, \xi_{2}}$ is $\left(m^{2}+n^{2}+2\right)(g-1)+2$, where $g$ is the genus of $X$.

In fact we compute the infinitesimal deformations of $S_{\lambda}^{\xi_{1}, \xi_{2}}$ explicitly.

The infinitesimal deformations of Calabi-Eckmann manifolds were computed by Akao in [1].

\section{Generalized Calabi-Eckmann manifolds}

We briefly recall the construction of the Calabi-Eckmann manifolds (see [2]). Take integers $m, n \geq 2$, and take $\lambda \in \mathbf{C}$ with $\operatorname{Im} \lambda>0$. Consider $\left(\mathbf{C}^{m} \backslash\{0\}\right) \times\left(\mathbf{C}^{n} \backslash\{0\}\right)$. The additive group $\mathbf{C}$ acts on this product as follows:

$$
t \cdot(z, w)=(\exp (t) z, \exp (t(\lambda-1) / \lambda) w), t \in \mathbf{C},(z, w) \in\left(\mathbf{C}^{m} \backslash\{0\}\right) \times\left(\mathbf{C}^{n} \backslash\{0\}\right) .
$$

The quotient

$$
M_{\lambda}^{m, n}:=\left(\left(\mathbf{C}^{m} \backslash\{0\}\right) \times\left(\mathbf{C}^{n} \backslash\{0\}\right)\right) / \mathbf{C}
$$

is a Calabi-Eckmann manifold. Let $S^{2 m-1}$ and $S^{2 n-1}$ be the unit spheres in $\mathbf{C}^{m}$ and $\mathbf{C}^{n}$ respectively. The composition of maps

$$
S^{2 m-1} \times S^{2 n-1} \hookrightarrow\left(\mathbf{C}^{m} \backslash\{0\}\right) \times\left(\mathbf{C}^{n} \backslash\{0\}\right) \longrightarrow M_{\lambda}^{m, n}
$$

is a diffeomorphism. Let

$$
T_{\lambda}:=\mathbf{C} /(\mathbf{Z} \oplus \lambda \cdot \mathbf{Z})
$$

be the complex elliptic curve. The natural projection

$$
\left(\mathbf{C}^{m} \backslash\{0\}\right) \times\left(\mathbf{C}^{n} \backslash\{0\}\right) \longrightarrow \mathbf{C} \mathbf{P}^{m-1} \times \mathbf{C P}^{n-1}
$$

descends to a projection to $\mathbf{C} \mathbf{P}^{m-1} \times \mathbf{C} \mathbf{P}^{n-1}$ of the above quotient space $M_{\lambda}^{m, n}$. This projection $M_{\lambda}^{m, n} \longrightarrow \mathbf{C} \mathbf{P}^{m-1} \times \mathbf{C P}^{n-1}$ makes $M_{\lambda}^{m, n}$ a holomorphic principal $T_{\lambda}$-bundle over $\mathbf{C P}^{m-1} \times$ $\mathbf{C} \mathbf{P}^{n-1}$. We will extend this construction to a family parametrized by a Riemann surface. 
Let $X$ be a compact connected Riemann surface of genus $g$. Let

$$
\xi_{1}: E_{1} \longrightarrow X \quad \text { and } \quad \xi_{2}: E_{2} \longrightarrow X
$$

be two holomorphic vector bundles over $X$ of rank $m$ and $n$ respectively; as before, $m, n \geq 2$. Let $E_{i}^{0}, i=1,2$, be the complement of the image of the zero section in the total space of $E_{i}$. Take $\lambda \in \mathbf{C}$ as above. The additive group $\mathbf{C}$ acts on the fiber product $E_{1}^{0} \times_{X} E_{2}^{0}$ as follow:

$$
t \cdot(z, w)=(\exp (t) \cdot z, \exp (t(\lambda-1) / \lambda) \cdot w), \quad t \in \mathbf{C},(z, w) \in E_{1}^{0} \times_{X} E_{2}^{0} .
$$

It is easy to check that this $\mathbf{C}$-action is free and proper. Hence the corresponding quotient

$$
S_{\lambda}^{\xi_{1}, \xi_{2}}:=\left(E_{1}^{0} \times_{X} E_{2}^{0}\right) / \mathbf{C}
$$

is a compact complex manifold (see, for example, [5, Proposition 2.1.13]). The projection $\left.\left(\xi_{1}, \xi_{2}\right)\right|_{E_{1}^{0} \times E_{2}^{0}}: E_{1}^{0} \times E_{2}^{0} \longrightarrow X$ descends to a holomorphic projection

$$
p: S_{\lambda}^{\xi_{1}, \xi_{2}} \longrightarrow X
$$

This projection makes $S_{\lambda}^{\xi_{1}, \xi_{2}}$ a holomorphic fiber bundle over $X$ with fiber $M_{\lambda}^{m, n}$ (constructed in (2.1)). The complex manifold $M_{\lambda}^{m, n}$ is not Kähler because $H^{2}\left(M_{\lambda}^{m, n}, \mathbf{R}\right)=0$. Hence $S_{\lambda}^{\xi_{1}, \xi_{2}}$ is also not Kähler (any complex submanifold of a Kähler manifold is Kähler).

For $i=1,2$, let $P\left(E_{i}\right)$ be the holomorphic projective bundles over $X$ parametrizing all the lines in $E_{i}$. The natural projection of $E_{1}^{0} \times_{X} E_{2}^{0}$ to $P\left(E_{1}\right) \times_{X} P\left(E_{2}\right)$ descends to a projection

$$
\varphi: S_{\lambda}^{\xi_{1}, \xi_{2}} \longrightarrow P\left(E_{1}\right) \times_{X} P\left(E_{2}\right) .
$$

We note that $P\left(E_{1}\right) \times_{X} P\left(E_{2}\right)$ is a complex projective manifold. The projection $p$ in (2.4) is the composition of $\varphi$ with the natural projection

$$
q: P\left(E_{1}\right) \times_{X} P\left(E_{2}\right) \longrightarrow X .
$$

The projection $\varphi$ makes $S_{\lambda}^{\xi_{1}, \xi_{2}}$ a holomorphic principal $T_{\lambda}$ bundle over $P\left(E_{1}\right) \times_{X} P\left(E_{2}\right)$, where $T_{\lambda}$ is defined in (2.2). To see this, consider the action of the multiplicative group $\mathbf{C}^{*}=$ $\mathbf{C} /(2 \pi \sqrt{-1} \cdot \mathbf{Z})$ on $E_{1}^{0} \times_{X} E_{2}^{0}$ defined by $t \cdot(z, w)=(t \cdot z, t \cdot w)$. This action commutes with the above action of $\mathbf{C}$ on $E_{1}^{0} \times{ }_{X} E_{2}^{0}$. Therefore, we get an action of $\mathbf{C}^{*}$ on the quotient $S_{\lambda}^{\xi_{1}, \xi_{2}}$. This action of $\mathbf{C}^{*}$ on $S_{\lambda}^{\xi_{1}, \xi_{2}}$ factors through the quotient group $T_{\lambda}=\mathbf{C}^{*} /\langle\exp (2 \pi \sqrt{-1} \cdot \lambda)\rangle$. Using this action of $T_{\lambda}$, the projection $\varphi$ is a holomorphic principal $T_{\lambda}$-bundle over $P\left(E_{1}\right) \times{ }_{X} P\left(E_{2}\right)$.

Fix Hermitian structures $h_{1}$ and $h_{2}$ on the vector bundles $E_{1}$ and $E_{2}$ respectively. Let

$$
S\left(\xi_{1}\right):=\left\{v \in E_{1} \mid h_{1}(v)=1\right\} \quad \text { and } \quad S\left(\xi_{2}\right):=\left\{v \in E_{2} \mid h_{2}(v)=1\right\}
$$

be the corresponding unit sphere bundles over $X$. Let

$$
S\left(\xi_{i}\right) \longrightarrow P\left(E_{i}\right)=E_{i}^{0} / \mathbf{C}^{*}
$$


be the restriction of the quotient map $E_{i}^{0} \longrightarrow P\left(E_{i}\right)$. It makes $S\left(\xi_{i}\right)$ a principal $S^{1}$-bundle over $P\left(E_{i}\right)$ (in particular, $S\left(\xi_{i}\right)$ is a circle bundle over $P\left(E_{i}\right)$ ). The composition of maps

$$
S\left(\xi_{1}\right) \times_{X} S\left(\xi_{2}\right) \hookrightarrow E_{1}^{0} \times_{X} E_{2}^{0} \longrightarrow S_{\lambda}^{\xi_{1}, \xi_{2}}
$$

is a diffeomorphism of fiber bundles over $X$. The complex structure on $S_{\lambda}^{\xi_{1}, \xi_{2}}$ produces a complex structure on $S\left(\xi_{1}\right) \times_{X} S\left(\xi_{2}\right)$ using this diffeomorphism.

\section{The Picard group}

For notational conveniences, $T_{\lambda}, M_{\lambda}^{m, n}$ and $S_{\lambda}^{\xi_{1}, \xi_{2}}$ will be denoted by $T, M$ and $S$ respectively. The fiber product $P\left(E_{1}\right) \times{ }_{X} P\left(E_{2}\right)$ will be denoted by $Y$.

Fix a point of $S$. Let $i: T \hookrightarrow S$ be the orbit of this point (recall that $S$ is a principal $T$-bundle over $Y$ ).

Proposition 3.1. Let $T \stackrel{i}{\hookrightarrow} S \stackrel{\varphi}{\longrightarrow} Y$ be the principal bundle in (2.5). Then we have the following short exact sequence:

$$
0 \longrightarrow H^{1}\left(Y, \mathcal{O}_{Y}\right) \stackrel{\varphi^{*}}{\longrightarrow} H^{1}\left(S, \mathcal{O}_{S}\right) \stackrel{i^{*}}{\longrightarrow} H^{1}\left(T, \mathcal{O}_{T}\right) \longrightarrow 0
$$

where $\varphi^{*}$ and $i^{*}$ are induced homomorphisms of cohomologies.

Proof. Consider the Borel spectral sequence (see Appendix 2 (page 202) of [4]) associated with the above principal bundle

$$
T \stackrel{i}{\hookrightarrow} S \stackrel{\varphi}{\longrightarrow} Y
$$

for the trivial holomorphic line bundle over $Y$. We have

$$
\begin{aligned}
& { }^{0,1} E_{2}^{1,0} \longrightarrow d_{2}{ }^{0,2} E_{2}^{3,-1}=0 \\
& { }^{0,1} E_{2}^{0,1} \longrightarrow d_{2}{ }^{0,2} E_{2}^{2,0}=H^{0,2}\left(Y, \mathcal{O}_{Y}\right) .
\end{aligned}
$$

From the Leray-Hirsch theorem for the fiber bundle in (2.6) it follows that the cohomology algebra $H^{*}(Y, \mathbf{C})$ is generated by $H^{2}(X, \mathbf{C})$ together with $c_{1}\left(\mathcal{O}_{P\left(E_{1}\right)}\right)$ and $c_{1}\left(\mathcal{O}_{P\left(E_{2}\right)}\right)$ (see [3, p. 432, Theorem 4D.1] for the Leray-Hirsch theorem). Therefore, $H^{2}(Y, \mathbf{C})=H^{1,1}(Y)$. In other words, $H^{0,2}\left(Y, \mathcal{O}_{Y}\right)=0$.

As no element of ${ }^{0,1} E_{r}^{1,0}$ and ${ }^{0,1} E_{r}^{0,1}$ is $d_{r}$-boundary for $r \geq 2$, we have ${ }^{0,1} E_{2}^{1,0}=$ ${ }^{0,1} E_{\infty}^{1,0}$ and ${ }^{0,1} E_{2}^{0,1}={ }^{0,1} E_{\infty}^{0,1}$. We have a filtration

$$
H^{1}\left(S, \mathcal{O}_{S}\right)=D^{1} \supset D^{0} \supset 0
$$

where $D^{0}={ }^{0,1} E_{\infty}^{1,0}$ and $D^{1} / D^{0}={ }^{0,1} E_{\infty}^{0,1}$. The corresponding graded object is

$$
\operatorname{Gr} H^{1}\left(S, \mathcal{O}_{S}\right)={ }^{0,1} E_{\infty}^{1,0} \oplus{ }^{0,1} E_{\infty}^{0,1} .
$$


Hence, the natural homomorphism

$$
\varphi^{*}: H^{1}\left(Y, \mathcal{O}_{Y}\right)={ }^{0,1} E_{2}^{1,0} \longrightarrow{ }^{0,1} E_{\infty}^{1,0}=D^{0} \subseteq H^{1}\left(S, \mathcal{O}_{S}\right)
$$

is injective, and the natural homomorphism

$$
i^{*}: H^{1}\left(S, \mathcal{O}_{S}\right)=D^{1} \longrightarrow D^{1} / D^{0}={ }^{0,1} E_{\infty}^{0,1}={ }^{0,1} E_{2}^{0,1}=H^{1}(T, \mathcal{O})
$$

is surjective. So we have the exact sequence

$$
0 \longrightarrow H^{1}\left(Y, \mathcal{O}_{Y}\right) \stackrel{\varphi^{*}}{\longrightarrow} H^{1}\left(S, \mathcal{O}_{S}\right) \stackrel{i^{*}}{\longrightarrow} H^{1}\left(T, \mathcal{O}_{T}\right) \longrightarrow 0 .
$$

This completes the proof.

LEMMA 3.2. For the projection $q$ in (2.6), the homomorphism

$$
q^{*}: H^{1}\left(X, \mathcal{O}_{X}\right) \longrightarrow H^{1}\left(Y, \mathcal{O}_{Y}\right)
$$

is an isomorphism. In particular, $\operatorname{dim} H^{1}\left(Y, \mathcal{O}_{Y}\right)=g$.

Proof. Since the fibers of $q$ are connected and simply connected, the long exact sequence of homotopy groups for $q$ gives that the homomorphism $\pi_{1}(Y) \longrightarrow \pi_{1}(X)$ induced by $q$ is an isomorphism. Hence $q^{*}: H^{1}(X, \mathbf{Q}) \longrightarrow H^{1}(Y, \mathbf{Q})$ is an isomorphism. Since both $X$ and $Y$ are Kähler, this implies the lemma.

Proposition 3.1 and Lemma 3.2 together have the following corollary:

COROLlary 3.3. The dimension of $H^{1}\left(S, \mathcal{O}_{S}\right)$ is $g+1$.

LEMMA 3.4. For the projection $p$ in (2.4), the homomorphism

$$
p_{*}: \pi_{1}(S) \longrightarrow \pi_{1}(X)
$$

is an isomorphism. In particular, the pullback homomorphism

$$
p^{*}: H^{1}(X, \mathbf{Z}) \longrightarrow H^{1}(S, \mathbf{Z})
$$

is an isomorphism.

PROOF. The fiber $M_{\lambda}^{m, n}$ of $p$ is connected and simply connected (it is a product of two spheres of dimensions at least three). Hence from the homotopy exact sequence it follows that the above homomorphism $p_{*}$ is an isomorphism. Therefore, the homomorphism

$$
H_{1}(S, \mathbf{Z}) \longrightarrow H_{1}(X, \mathbf{Z})
$$

given by $p$ is an isomorphism. Now from the universal coefficient theorem for cohomologies it follows that the homomorphism $p^{*}$ in the lemma is an isomorphism. 
PROPOSITION 3.5. The pullback homomorphism

$$
p^{*}: H^{2}(X, \mathbf{Z}) \longrightarrow H^{2}(S, \mathbf{Z})
$$

is an isomorphism.

PROOF. Let

$$
M \stackrel{\iota}{\hookrightarrow} S \stackrel{p}{\longrightarrow} X
$$

be the fiber bundle in (2.4). Consider the Serre spectral sequence associated to this fiber bundle for the constant sheaf $\mathbf{Z}$. We will show that the local system $R^{i} p_{*} \mathbf{Z}$ is constant for all $i$. Recall that the fibers of $p$ are $M=S^{2 m-1} \times S^{2 n-1}$. For the action of $\mathrm{U}(m)$ on $S^{2 m-1}=\left\{v \in \mathbf{C}^{m} \mid\|v\|^{2}=1\right\}$, the action of $\mathrm{U}(m)$ on $H^{*}\left(S^{2 m-1}, \mathbf{Z}\right)$ is trivial. Similarly, $\mathrm{U}(n)$ acts trivially on $H^{*}\left(S^{2 n-1}, \mathbf{Z}\right)$. Therefore, the local system $R^{i} p_{*} \mathbf{Z}$ is constant for all $i$.

Consequently, we have

$$
\begin{aligned}
& E_{2}^{0,2}=H^{0}(X, \mathbf{Z}) \otimes H^{2}(M, \mathbf{Z})=0, \\
& E_{2}^{1,1}=H^{1}(X, \mathbf{Z}) \otimes H^{1}(M, \mathbf{Z})=0, \\
& E_{2}^{2,0}=H^{2}(X, \mathbf{Z}) \otimes H^{0}(M, \mathbf{Z})=H^{2}(X, \mathbf{Z}) .
\end{aligned}
$$

Further,

$$
d_{2}: E_{2}^{0,1}=0 \longrightarrow E_{2}^{2.0}
$$

is a zero map. This implies that

$$
E_{\infty}^{0,2}=0, E_{\infty}^{1,1}=0 \text { and } E_{\infty}^{2,0}=H^{2}(X, \mathbf{Z})
$$

Hence the pullback homomorphism

$$
p^{*}: H^{2}(X, \mathbf{Z})=E_{\infty}^{2,0} \longrightarrow H^{2}(S, \mathbf{Z})
$$

is an isomorphism.

THEOREM 3.6. The Picard group of S fits in a short exact sequence

$$
0 \longrightarrow \operatorname{Pic}(X) \longrightarrow \operatorname{Pic}(S) \longrightarrow H^{1}\left(T, \mathcal{O}_{T}\right) \longrightarrow 0 \text {. }
$$

The injective homomorphism $\operatorname{Pic}(X) \longrightarrow \operatorname{Pic}(S)$ sends any holomorphic line bundle $L$ to $p^{*} L$.

Proof. Let $\mathcal{O}_{S}^{*}$ be the multiplicative sheaf on $S$ of nowhere zero holomorphic functions. Consider the following short exact sequence of sheaves on $S$

$$
0 \longrightarrow \mathbf{Z} \longrightarrow \mathcal{O}_{S} \longrightarrow \mathcal{O}_{S}^{*} \longrightarrow 0,
$$


where the surjective homomorphism is $f \longmapsto \exp (2 \pi \sqrt{-1} \cdot f)$. From the long exact sequence of cohomologies associated to it we conclude that $\operatorname{Pic}(S)$ fits in the exact sequence

$$
H^{1}(S, \mathbf{Z}) \longrightarrow H^{1}\left(S, \mathcal{O}_{S}\right) \longrightarrow \operatorname{Pic}(S) \longrightarrow H^{2}(S, \mathbf{Z}) \longrightarrow H^{1}\left(S, \mathcal{O}_{S}\right)
$$

We have the exact sequence

$$
H^{1}(X, \mathbf{Z}) \longrightarrow H^{1}\left(X, \mathcal{O}_{X}\right) \longrightarrow \operatorname{Pic}(X) \longrightarrow H^{2}(X, \mathbf{Z}) \longrightarrow 0
$$

which is constructed from the short exact sequence

$$
0 \longrightarrow \mathbf{Z} \longrightarrow \mathcal{O}_{X} \longrightarrow \mathcal{O}_{X}^{*} \longrightarrow 0
$$

on $X$.

Consider the pullback homomorphism $p^{*}: \operatorname{Pic}(X) \longrightarrow \operatorname{Pic}(S)$ defined by $L \longmapsto$ $p^{*} L$. Since $H^{1}\left(X, \mathcal{O}_{X}\right) \subset H^{1}\left(S, \mathcal{O}_{S}\right)$ (see Proposition 3.1 and Lemma 3.2), $H^{1}(S, \mathbf{Z})=$ $H^{1}(X, \mathbf{Z})$ (see Lemma 3.4) and $H^{2}(S, \mathbf{Z})=H^{2}(X, \mathbf{Z})$ (see Proposition 3.5) with the homomorphisms given by pullback, we conclude from the above two exact sequences that the homomorphism $p^{*}$ makes $\operatorname{Pic}(X)$ a subgroup of $\operatorname{Pic}(S)$. Since $H^{1}\left(S, \mathcal{O}_{S}\right) / H^{1}\left(X, \mathcal{O}_{X}\right)=$ $H^{1}\left(T, \mathcal{O}_{T}\right)$ by Proposition 3.1 and Lemma 3.2, we conclude that $\operatorname{Pic}(S) / p^{*}(\operatorname{Pic}(X))=$ $H^{1}\left(T, \mathcal{O}_{T}\right)$. (The argument is same as the proof of five lemma.)

\section{Infinitesimal deformations of the complex structure}

In this section, we make the following assumptions:

(1) The two holomorphic vector bundles $E_{1}$ and $E_{2}$ are simple, meaning

$$
H^{0}\left(X, \operatorname{End}\left(E_{1}\right)\right)=\mathbf{C}=H^{0}\left(X, \operatorname{End}\left(E_{2}\right)\right) .
$$

(2) $\operatorname{genus}(X)=g \geq 2$.

We note that any stable holomorphic vector bundle is simple.

LeMmA 4.1. Let $\theta_{Y}$ be the holomorphic tangent bundles of $Y=P\left(E_{1}\right) \times_{X} P\left(E_{2}\right)$. Then $H^{0}\left(Y, \theta_{Y}\right)=0$.

Proof. For $i=1,2$, let ad $\left(E_{i}\right) \subset \operatorname{End}\left(E_{i}\right)$ be the holomorphic subbundle of co-rank one defined by the sheaf of endomorphisms of $E_{i}$ of trace zero. So, $\operatorname{End}\left(E_{i}\right)=\operatorname{ad}\left(E_{i}\right) \oplus \mathcal{O}_{X}$. We note that

$$
H^{0}\left(X, \operatorname{ad}\left(E_{i}\right)\right)=0
$$

because $E_{i}$ is simple.

Consider the projection $q$ in (2.6). Let $\theta_{Y / X} \subset \theta_{Y}$ be the relative holomorphic tangent bundle for $q$. We note that

$$
q_{*} \theta_{Y / X}=\operatorname{ad}\left(E_{1}\right) \oplus \operatorname{ad}\left(E_{2}\right) .
$$


The short exact sequence of holomorphic vector bundles

$$
0 \longrightarrow \theta_{Y / X} \longrightarrow \theta_{Y} \longrightarrow q^{*} \theta_{X} \longrightarrow 0,
$$

where $\theta_{X}$ is the holomorphic tangent bundle of $X$, produces a short exact sequence

$$
0 \longrightarrow q_{*} \theta_{Y / X} \longrightarrow q_{*} \theta_{Y} \longrightarrow \theta_{X} \longrightarrow 0
$$

on $X$ because $R^{1} q_{*} \theta_{Y / X}=0$.

From (4.1) and (4.2) it follows that $H^{0}\left(X, q_{*} \theta_{Y / X}\right)=0$. We also have $H^{0}\left(X, \theta_{X}\right)=0$ because $g \geq 2$. Therefore, from the long exact sequence of cohomologies associated to (4.4) it follows that $H^{0}\left(X, q_{*} \theta_{Y}\right)=0$. This implies that $H^{0}\left(Y, \theta_{Y}\right)=0$.

LEMMA 4.2. The cohomology $H^{1}\left(Y, \theta_{Y}\right)$ fits in a natural short exact sequence

$$
0 \longrightarrow H^{1}\left(X, \operatorname{ad}\left(E_{1}\right)\right) \oplus H^{1}\left(X, \operatorname{ad}\left(E_{2}\right)\right) \longrightarrow H^{1}\left(Y, \theta_{Y}\right) \longrightarrow H^{1}\left(X, \theta_{X}\right) \longrightarrow 0 .
$$

Proof. Consider the short exact sequence in (4.3). We note that $R^{i} q_{*} \theta_{Y / X}=0$ for all $i \geq 1$. From the projection formula, and the fact that $H^{i}\left(\mathbf{C P}^{N}, \mathcal{O}_{\mathbf{C P}^{N}}\right)=0$ for all $i \geq 1$ and all $N$, we conclude that

$$
R^{i} q_{*} q^{*} \theta_{X}=\theta_{X} \otimes R^{i} q_{*} \mathcal{O}_{Y}=0
$$

for all $i \geq 1$. Therefore,

$$
H^{j}\left(Y, \theta_{Y / X}\right)=H^{j}\left(X, q_{*} \theta_{Y / X}\right)=H^{j}\left(X, \operatorname{ad}\left(E_{1}\right) \oplus \operatorname{ad}\left(E_{2}\right)\right)
$$

(see (4.2) for the second equality) and

$$
H^{j}\left(Y, q^{*} \theta_{X}\right)=H^{j}\left(X, q_{*} q^{*} \theta_{X}\right)=H^{j}\left(X, \theta_{X}\right)
$$

for all $j \geq 0$. In particular, $H^{0}\left(Y, q^{*} \theta_{X}\right)=H^{0}\left(X, \theta_{X}\right)=0$ (because $g \geq 2$ ), and $H^{2}\left(Y, \theta_{Y / X}\right)=H^{2}\left(X, q_{*} \theta_{Y / X}\right)=0$. Therefore, the long exact sequence of cohomologies for (4.3) gives the short exact sequence

$$
\begin{gathered}
0 \longrightarrow H^{1}\left(Y, \theta_{Y / X}\right)=H^{1}\left(X, \operatorname{ad}\left(E_{1}\right) \oplus \operatorname{ad}\left(E_{2}\right)\right) \longrightarrow H^{1}\left(Y, \theta_{Y}\right) \\
\longrightarrow H^{1}\left(Y, q^{*} \theta_{X}\right)=H^{1}\left(X, \theta_{X}\right) \longrightarrow 0 .
\end{gathered}
$$

From this the lemma follows because $H^{1}\left(X, \operatorname{ad}\left(E_{1}\right) \oplus \operatorname{ad}\left(E_{2}\right)\right)=H^{1}\left(X, \operatorname{ad}\left(E_{1}\right)\right) \oplus$ $H^{1}\left(X, \operatorname{ad}\left(E_{2}\right)\right)$.

Proposition 4.3. Let $\theta_{S}$ be the holomorphic tangent bundles of $S$. Then $H^{0}\left(S, \theta_{S}\right)=\mathbf{C}$.

The cohomology $H^{1}\left(S, \theta_{S}\right)$ fits in a natural short exact sequence

$$
0 \longrightarrow H^{1}\left(S, \mathcal{O}_{S}\right) \longrightarrow H^{1}\left(S, \theta_{S}\right) \longrightarrow H^{1}\left(Y, \theta_{Y}\right) \longrightarrow 0
$$


Proof. Consider the Borel spectral sequence associated to $\varphi$ for the tangent bundle $T Y$. We have

$$
{ }^{0,0} E_{\infty}^{0,0}={ }^{0,0} E_{2}^{0,0}=H^{0}\left(Y, \theta_{Y}\right) .
$$

Now, Lemma 4.1 says that $H^{0}\left(Y, \theta_{Y}\right)=0$. Hence

$$
H^{0}\left(S, \varphi^{*} \theta_{Y}\right)={ }^{0,0} E_{\infty}^{0,0}=0 .
$$

Let $\theta_{S / Y} \subset \theta_{S}$ be the relative tangent bundle for the projection $\varphi$. We note that $\theta_{S / Y}=$ $\mathcal{O}_{S}$ using the action of $T$ on $S$. Consider the long exact sequence of cohomologies associated to the short exact sequence of vector bundles

$$
0 \longrightarrow \theta_{S / Y}=\mathcal{O}_{S} \longrightarrow \theta_{S} \longrightarrow \varphi^{*} \theta_{Y} \longrightarrow 0 .
$$

Since $H^{0}\left(S, \varphi^{*} \theta_{Y}\right)=0$, we conclude that the homomorphism

$$
H^{0}\left(S, \theta_{S / Y}\right)=H^{0}\left(S, \mathcal{O}_{S}\right) \longrightarrow H^{0}\left(S, \theta_{S}\right)
$$

in the long exact sequence is an isomorphism. Therefore, the first statement of the proposition is proved.

To prove the second part of the proposition, first note that

$$
{ }^{0,1} E_{2}^{0,1}=H^{0,0}\left(Y, \theta_{Y}\right) \otimes H^{0,1}\left(T, \mathcal{O}_{T}\right)=0
$$

because $H^{0}\left(Y, \theta_{Y}\right)=0$. Hence ${ }^{0,1} E_{\infty}^{0,1}=0$. Further, since

$$
{ }^{0,1} E_{2}^{1,0}=H^{0,1}\left(Y, \theta_{Y}\right) \stackrel{d_{2}}{\longrightarrow}{ }^{0,2} E_{2}^{3,-1}=0,
$$

we conclude that ${ }^{0,1} E_{\infty}^{1,0}=H^{0,1}\left(Y, \theta_{Y}\right)$.

Now, let

$$
H^{1}\left(S, \varphi^{*} \theta_{Y}\right)=D^{1} \supset D^{0} \supset 0
$$

be the natural filtration for which the corresponding graded object is

$$
\operatorname{Gr} H^{1}\left(S, \varphi^{*} \theta_{X}\right)={ }^{0,1} E_{\infty}^{1,0} \oplus{ }^{0,1} E_{\infty}^{0,1},
$$

more precisely, $D^{0}={ }^{0,1} E_{\infty}^{1,0}$ and $D^{1} / D^{0}={ }^{0,1} E_{\infty}^{0,1}$. Since ${ }^{0,1} E_{\infty}^{0,1}=0$, we have $D^{1}=D^{0}$. This implies that the natural homomorphism

$$
\varphi^{*}: H^{1}\left(Y, \theta_{Y}\right)={ }^{0,1} E_{2}^{1,0} \longrightarrow{ }^{0,1} E_{\infty}^{1,0}=D^{0}=D^{1}=H^{1}\left(S, \varphi^{*} \theta_{Y}\right)
$$

is an isomorphism.

Consider the long exact sequence of cohomologies

$$
H^{0}\left(S, \varphi^{*} \theta_{Y}\right) \longrightarrow H^{1}\left(S, \mathcal{O}_{S}\right) \longrightarrow H^{1}\left(S, \theta_{S}\right) \stackrel{\phi}{\longrightarrow} H^{1}\left(S, \varphi^{*} \theta_{Y}\right)
$$


associated to the short exact sequence in (4.6). Since $H^{0}\left(S, \varphi^{*} \theta_{Y}\right)=0$ (see (4.5)) and $H^{1}\left(S, \varphi^{*} \theta_{Y}\right)=H^{1}\left(Y, \theta_{Y}\right)$ (see (4.7)), to prove the second part of the proposition it suffices to show that the homomorphism $\phi$ in (4.8) is surjective.

From Lemma 4.2 we know that all the infinitesimal deformations of $Y$ are given by the infinitesimal deformations of the two vector bundles $E_{1}$ and $E_{2}$ and the infinitesimal deformations of the Riemann surface $X$. The subspaces

$$
H^{1}\left(X, \operatorname{ad}\left(E_{1}\right)\right) \subset H^{1}\left(Y, \theta_{Y}\right) \text { and } H^{1}\left(X, \operatorname{ad}\left(E_{2}\right)\right) \subset H^{1}\left(Y, \theta_{Y}\right)
$$

in Lemma 4.2 correspond to the infinitesimal deformations of the projective bundle $P\left(E_{1}\right)$ and $P\left(E_{2}\right)$ respectively (keeping the Riemann surface $X$ fixed). The infinitesimal deformations of $E_{1}$ (respectively, $\left.E_{2}\right)$ is given by $H^{1}\left(X, \operatorname{End}\left(E_{1}\right)\right)$ (respectively, $H^{1}\left(X, \operatorname{End}\left(E_{2}\right)\right)$ ). The natural map from the infinitesimal deformations of $E_{i}$ to the infinitesimal deformations of $P\left(E_{i}\right)$ corresponds to the projection $H^{1}\left(X, E n d\left(E_{i}\right)\right) \longrightarrow H^{1}\left(X, \operatorname{ad}\left(E_{i}\right)\right)$ given by the decomposition $\operatorname{End}\left(E_{i}\right)=\operatorname{ad}\left(E_{i}\right) \oplus \mathcal{O}_{X}$. The projection $H^{1}\left(Y, \theta_{Y}\right) \longrightarrow H^{1}\left(X, \theta_{X}\right)$ corresponds to the infinitesimal deformations of $X$. All these infinitesimal deformations give rise to infinitesimal deformations of $S$. Hence the homomorphism $\phi$ in (4.8) is surjective.

COROLlary 4.4. The dimension of $H^{1}\left(S, \theta_{S}\right)$ is $\left(m^{2}+n^{2}+2\right)(g-1)+2$.

Proof. Since $H^{0}\left(X, \operatorname{ad}\left(E_{1}\right)\right)=0=H^{0}\left(X, \operatorname{ad}\left(E_{2}\right)\right)$ (recall that $E_{1}$ and $E_{2}$ are both simple), from the Riemann-Roch theorem we have

$$
\operatorname{dim} H^{1}\left(X, \operatorname{ad}\left(E_{1}\right)\right)=\left(m^{2}-1\right)(g-1) \text { and } \operatorname{dim} H^{1}\left(X, \operatorname{ad}\left(E_{2}\right)\right)=\left(n^{2}-1\right)(g-1) .
$$

Therefore, Proposition 4.3 and Lemma 4.2,

$$
\operatorname{dim} H^{1}\left(S, \theta_{S}\right)=\left(m^{2}+n^{2}+1\right)(g-1)+\operatorname{dim} H^{1}\left(S, \mathcal{O}_{S}\right) .
$$

Now the corollary follows from Corollary 3.3.

\section{Computation of the Brauer group}

Let $M$ be a compact connected complex manifold. Let $\mathcal{O}_{M}^{*}$ be the multiplicative sheaf on $M$ of nowhere zero holomorphic functions. The cohomological Brauer group $\operatorname{Br}^{\prime}(M)$ is the group of torsion elements in $H^{2}\left(M, \mathcal{O}_{M}^{*}\right)$.

To define the Brauer group of $M$, consider all holomorphic principal PGL $(r, \mathbf{C})$-bundles on $M$ for all $r \geq 1$. Let

$$
\mathrm{GL}(r, \mathbf{C}) \times \mathrm{GL}\left(r^{\prime}, \mathbf{C}\right) \longrightarrow \mathrm{GL}\left(r r^{\prime}, \mathbf{C}\right)
$$

be the homomorphism given by the natural action of any $A \times B \in \operatorname{GL}(r, \mathbf{C}) \times \operatorname{GL}\left(r^{\prime}, \mathbf{C}\right)$ on $\mathbf{C}^{r} \otimes \mathbf{C}^{r^{\prime}}$. This homomorphism descends to a homomorphism

$$
\gamma: \operatorname{PGL}(r, \mathbf{C}) \times \operatorname{PGL}\left(r^{\prime}, \mathbf{C}\right) \longrightarrow \operatorname{PGL}\left(r r^{\prime}, \mathbf{C}\right) .
$$


Given a holomorphic principal $\operatorname{PGL}(r, \mathbf{C})$-bundle $\mathcal{A}$ on $M$ and a holomorphic principal $\operatorname{PGL}\left(r^{\prime}, \mathbf{C}\right)-$ bundle $\mathcal{B}$ on $M$, the homomorphism $\gamma$ produces a holomorphic principal $\operatorname{PGL}\left(r r^{\prime}, \mathbf{C}\right)$-bundle on $M$ by extension of structure group. This holomorphic principal PGL $\left(r r^{\prime}, \mathbf{C}\right)-$ bundle will be denoted by $\mathcal{A} \otimes \mathcal{B}$. The two principal bundles $\mathcal{A}$ and $\mathcal{B}$ will be called equivalent if there are holomorphic vector bundles $V$ and $W$ on $M$ such that $\mathcal{A} \otimes P(V)$ is holomorphically isomorphic to $\mathcal{B} \otimes P(W)$.

The equivalence classes of projective bundles form a group. The addition operation is given by the tensor product, and the inverse is given by the automorphism $A \longmapsto\left(A^{t}\right)^{-1}$ of $\operatorname{PGL}(r, \mathbf{C})$ (it corresponds to taking the dual projective bundles). (See [6, Section 1] for the details.) This group is called the Brauer group of $M$, and it is denoted by $\operatorname{Br}(M)$.

The Brauer group $\operatorname{Br}(M)$ is a subgroup of the cohomological Brauer group $\operatorname{Br}^{\prime}(M)[6$, p. 878].

Let $T$ denote the torsion part of $H^{3}(M, \mathbf{Z})$. Let

$$
\gamma: H^{1}\left(M, \mathcal{O}_{M}^{*}\right) \longrightarrow H^{2}(M, \mathbf{Z})
$$

be the homomorphism that sends any holomorphic line bundle on $M$ to its first Chern class. Let

$$
A:=H^{2}(M, \mathbf{Z}) / \gamma\left(H^{1}\left(M, \mathcal{O}_{M}^{*}\right)\right)
$$

be the quotient. The cohomological Brauer group $\operatorname{Br}^{\prime}(M)$ fits in a short exact sequence

$$
0 \longrightarrow A \otimes(\mathbf{Q} / \mathbf{Z}) \longrightarrow \operatorname{Br}^{\prime}(M) \longrightarrow T \longrightarrow 0
$$

(see [6, p. 878, Proposition 1.1]).

Proposition 5.1. Let $M \stackrel{\iota}{\hookrightarrow} S \stackrel{p}{\longrightarrow} X$ be the holomorphic fiber bundle in (2.4). Then the cohomology group $H^{3}(S, \mathbf{Z})$ is torsionfree.

PROOF. The proof is similar to the proof of Proposition 3.5. Consider the Serre spectral sequence associated to the fiber bundle

$$
M \stackrel{\iota}{\hookrightarrow} S \stackrel{p}{\longrightarrow} X
$$

for the constant sheaf $\mathbf{Z}$. We have seen in the proof of Proposition 3.5 that the local system $R^{i} p_{*} \mathbf{Z}$ is constant for all $i$.

We have

$$
\begin{aligned}
& E_{2}^{0,3}=H^{0}(X, \mathbf{Z}) \otimes H^{3}(M, \mathbf{Z})=H^{3}(M, \mathbf{Z}), \\
& E_{2}^{2,1}=H^{2}(X, \mathbf{Z}) \otimes H^{1}(M, \mathbf{Z})=0, \\
& E_{2}^{1,2}=H^{1}(X, \mathbf{Z}) \otimes H^{2}(M, \mathbf{Z})=0 \\
& E_{2}^{3,0}=H^{3}(X, \mathbf{Z}) \otimes H^{0}(M, \mathbf{Z})=0 .
\end{aligned}
$$


With a similar argument as above, we can conclude that

$$
H^{3}(S, \mathbf{Z})=E_{\infty}^{0,3}=E_{2}^{0,3}=H^{3}(M, \mathbf{Z}) .
$$

Since $M=S^{2 m-1} \times S^{2 n-1}$ with $m, n \geq 2$, it thus follows that $H^{3}(S, \mathbf{Z})$ is torsionfree.

COROLlary 5.2. The cohomological Brauer group $\mathrm{Br}^{\prime}(S)$ vanishes. The Brauer group $\operatorname{Br}(S)$ vanishes.

Proof. Every element of $H^{2}(X, \mathbf{Z})$ is the first Chern class of a holomorphic line bundle on $X$. Therefore, from Proposition 3.5 it follows that each element of $H^{2}(S, \mathbf{Z})$ is the first Chern class of a holomorphic line bundle on $S$. Now the first statement follows from (5.1) and Proposition 5.1. The second statement follows from the first statement because $\operatorname{Br}(S) \subset \operatorname{Br}^{\prime}(S)$.

\section{References}

[ 1] K. AKAO, On deformations of the Calabi-Eckmann manifolds, Proc. Japan Acad. 51 (1975), 365-368.

[2] E. CALABI and B. ECKMAnN, A class of compact, complex manifolds which are not algebraic, Ann. of Math. 58 (1953), 494-500.

[ 3 ] A. Hatcher, Algebraic topology, Cambridge University Press, 2002.

[4] F. Hirzebruch, Topological methods in algebraic geometry, Classics in Mathematics, Springer-Verlag, Berlin, 1995.

[ 5 ] D. HuYBRechts, Complex geometry. an introduction, Universitext, Springer-Verlag, Berlin, 2005.

[ 6 ] S. SCHRÖER, Topological methods for complex-analytic Brauer groups, Topology 44 (2005), 875-894.

Present Addresses:

INDRANIL BISWAS

SCHOOL OF MATHEMATICS,

TATa Institute of Fundamental Research, HOMI BHABHA ROAD, BOMBAY 400005, INDIA.

e-mail: indranil@math.tifr.res.in

MAHAN MJ

RKM VIVEKANANDA UNIVERSITY, BELUR MATH, WB 711202, INDIA.

e-mail: mahan.mj@gmail.com; mahan@rkmvu.ac.in

AJAY SINGH THAKUR

SCHOOL OF MATHEMATICS,

TATA INSTITUTE OF FUNDAMENTAL RESEARCH, HOMI BHABHA ROAD, BOMBAY 400005, INDIA. e-mail: athakur@math.tifr.res.in 